\title{
Image Spectrometry and Copernicus data for Heritage Mapping in the Jarama Valley, Spain
}

\author{
Rejas Ayuga, Juan Gregorio a,c *, González Matesanz, Francisco Javier ${ }^{\text {b,c }}$, Sánchez-Ortiz, Pilar b,c \\ ${ }^{a}$ Technical University of Madrid (UPM), C/ Profesor Aranguren s/n, 28040 Madrid, Spain, juangregorio.rejas@upm.es \\ ${ }^{b}$ National Geographic Institute (IGN), Madrid, Spain, fjdmatesanz@fomento.es \\ ${ }^{c}$ Spanish Society of Cartography, Photogrammetry and Remote Sensing (SECFT), Spain, psanchezortiz@secft.es \\ *rejasaj@inta.es
}

\begin{abstract}
For millions of years, the Jarama Valley, located in the middle of Spain, has fluctuated physically and geomorphological, supposing over the centuries a crucial settlement for wildlife and different human cultures as an efficient route through South Europa. Currently, this scenario, which consist of a vast and intricate network of military installations due to the Battle of Jarama in the Spanish Civil War, mixed with incredible paleontological sites, Celtiberian cities and roman, are part of a great-protected natural and cultural classified as the Southeast Regional Park close to Madrid City.

The aim of this work is developing a geospatial methodology for the digital heritage mapping in the Jarama Valley using geomatics' technologies in situ, drones' data, image spectrometry and the Copernicus European program, both their active and passive sensors. Data from Sentinel 1, Sentinel 2 and airborne sensors have analysed according to remote sensing techniques to recognize the potential re-mains and to reconstruct the heritage landscapes of three test areas in the Jarama Valley. We have investigated the spectral characteristics of main biophysical parameters in the pattern recognition of manmade materials in several remote sensing scenes of the Jarama Valley. Spectral ranges from drones' data are used to validate data cubes from multisperspectral sensor ATM. Sentinel 1 polarimetric products and thermal anomalies have calculated in order to contrast evidences of buried remains and confirm land change detection over the last decades. First results and their consequences on the digital heritage mapping of the Jarama Valley are discussed, representing a first point of protection and an advance of a full cultural and natural heritage research project in this region.
\end{abstract}

Keywords: Heritage, Copernicus European Program, Image Spectrometry, Remote Sensing Cartography.

\section{Introduction}

Heritage mapping by new image technologies and nondestructive techniques out of which outstands remote sensing through different platforms (space, airborne and short range), represent an important tool (Rejas and Burillo, 2016; Rejas et al., 2009) for the improvement, protection and prevention of cultural and natural heritage. Since their early stages, remote sensing techniques have been applied to mapping using instruments that measure (usually covering a 2-D area and so rendering an image) the electromagnetic radiation coming from the study target. This radiation being either reflected sunlight, thermally emitted radiance or (in so-called active systems) energy sent by the instrument itself (microwaves in SAR or laser beam in a LIDAR).

In other sense, image spectrometry has proven to be efficient in the characterization of materials based on statistical methods using specific reflection and absorption bands (Nasrabadi, 2008; Rejas and Burillo, 2016). Spectral configurations in the Visible and Near Infrared (VNIR), Short Wave Infrared (SWIR), Thermal Infrared (TIR) and microwaves by Synthetic Aperture
Radar (SAR) have been successfully used for mapping materials in different heritage scenarios (Farjas et al., 2003; Rejas et al., 2009).

An important aim in this research is to establish relationships that allow linking spectral patterns with what can be called as real information and, therefore, identify heritage information related to anomalous responses rather than simply spotting differences from the natural background. We can mapping biophysical parameters using new remote sensing technologies as the European Copernicus program and calculate spectral, thermal and microwaves patterns that could be indicative of the presence of anthropomorphic structures in heritage sites or pathologies that affect these structures.

This work presents a multi-methodology approach applied to the analysis of correlations between spectral information and cultural heritage information in the Jarama Valley in Spain. The main finding in our study is that it is possible at all to just detect spectral patterns and then predict something about the heritage information and somehow our study improve how to extract it. In this sense, first results and their consequences on the digital mapping registration of the Jarama Valley are discussed, 
representing a first point of protection and an advance of a full cultural and natural heritage research project in this region.

\section{Regional Park of Southeast of Madrid. Data and Exploratory Analysis.}

For millions of years, the Jarama Valley, located in the middle of Spain, has fluctuated physically and geomorphological, supposing over the centuries a crucial settlement for wildlife and different human cultures as an efficient route through South Europa. Currently, this scenario, which consist of a vast and intricate network of military installations due to the Battle of Jarama in the Spanish Civil War, mixed with incredible paleontological sites, Celtiberian cities and roman, are part of a greatprotected natural and cultural classified as the Southeast Regional Park close to Madrid City

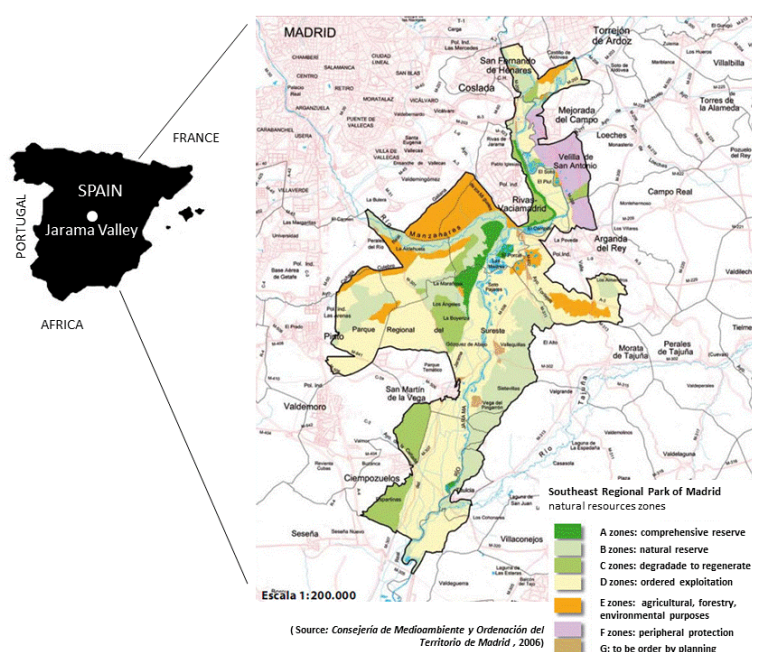

Figure 1. Location of the Southeast Regional Park of Madrid. (source: Consejería de Medioambiente y Ordenación del Territorio de la Comunidad de Madrid, 2006)

We have used for this case several images of Sentinel 1 and Sentinel 2 from the European program Copernicus, and scenes of the ATM (Airborne Thematic Mapper) sensor (Farjas et al., 2003) with the aim to detect heritage structure remains like settlements, ancient buildings, walls, trenches, bunkers or tunnels. Sentinel 1 is a single C-band synthetic aperture radar (https://sentinel.esa.int/web/sentinel/missions/sentinel1/instrument-payload) and Sentinel 2 is a highresolution multispectral instrument (https://www.esa.int/Our_Activities/Observing_the_Earth /Copernicus/Sentinel-2/Instrument).

ATM is an opto-mechanical radiometer with image formation through the combination of a spinning mirror and the aircraft motion. It split the incoming radiation in 11 spectral bands, from visible through near infrared up to thermal infrared, and with a spatial resolution of $3.4 \mathrm{~m}$.

Data pre-processing techniques are applied to extract information from remote sensing data. They usually include geometric correction, radiometric correction and a number of image arithmetic and statistical analysis. The choice of techniques depends on image quality and in the required output. A check of radiometric corrections of the images have been performed from data measured in field and laboratory with USB400 and ASD FieldSpec 4 HiRes spectroradiometers. The spectra have been used to characterize heritage materials and to check reflectance images by an empirical linear regression.

The airborne images have been georeferenced directly using the geometry calculated from the position and the attitude data measured by inertial GPS/IMU (Inertial Measurement Unit) at the same time of acquisition over the study area. The georeferencing images has been tested using check points measured on the ground in projection UTM and WGS84 Datum by DGPS (Differential Global Positioning System).

As exploratory analysis, vegetation indexes and soil have been calculated for the set of images. Several indexes of vegetation from imagery were generated, the Normalized Difference Vegetation Index (Rouse et al., 1974) for evaluating the estimate of the LAI cover (Leaf Area Index), and the ratio (Berni et al., 2010) between the rate of the Transformed Chlorophyll Absorption in Reflectance Index-TCARI (Haboudane et al. 2002) and the Optimized Soil-Adjusted Vegetation Index-OSAVI (Rondeaux et al., 1996).

$N D V I=\frac{\rho_{\mu N I R}-\rho_{\mu R}}{\rho_{\mu N I R}-\rho_{\mu R}}$

where $\rho_{\mu N I R}=$ near-infrared channel reflectance, $\rho_{\mu R}=$ red channel reflectance

TCARI / OSAVI

$=3 \cdot\left[\left(\rho_{\mu 700}-\rho_{\mu 670}\right)-0.2 \cdot\left(\rho_{\mu 700}-\rho_{\mu 550}\right) \cdot\left(\rho_{\mu 700}-\rho_{\mu 670}\right)\right]$

$(1+0.16) \cdot\left(\rho_{\mu 800}-\rho_{\mu 670}\right) /\left(\rho_{\mu 800}-\rho_{\mu 670}+0.16\right)$

where $\rho_{\mu i}=$ reflectance at wavelength

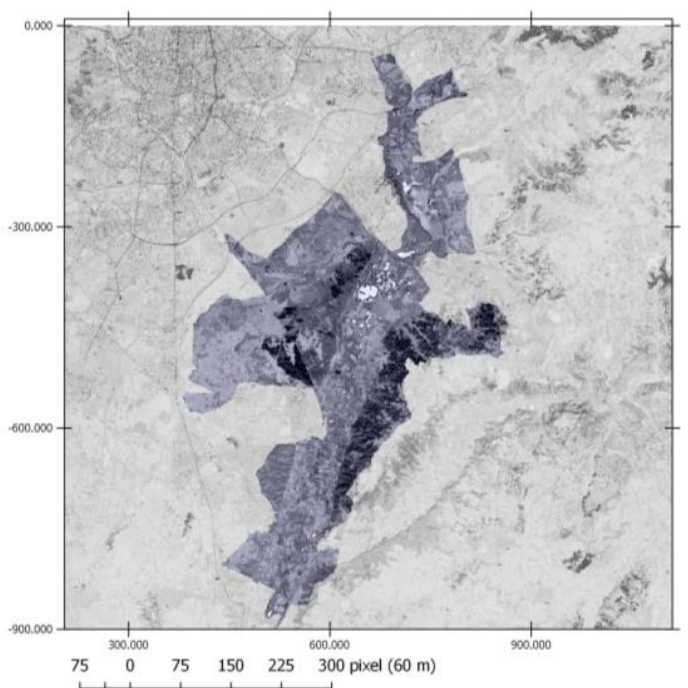

Figure 2. Example of TCARI index map (local coordinates in pixel relative to Madrid city) from Sentinel 2 of the Southeast Regional Park of Madrid.

\section{Data processing for heritage mapping}

Multi-source files have been generated in a way that an accurate spatial co-registration between the different 
pieces of information becomes crucial for the subsequent data analysis (Rejas and Burillo, 2016).

The integration of these results with SAR data has allowed the establishment of relationships between ground deformations, thermal variability and optimal paths.An interferometric Sentinel 1 pair from Sentinel 1 images of $6^{\text {th }}$ of November 2017 and 29 $9^{\text {th }}$ January 2018 has been processed. Currently, advanced SAR processed results are being analyzed and integrated into Geographic Information Systems, together with the remaining pieces of information that has been generated, with the aim of exploring the possibilities for its interconnection with possible regional park paths in the route along the Jarama Valley.

The main Southeast Regional Park heritage sites found between the East and West of the Jarama Valley have been recorded in the Sentinel 1 interferometric pair. Under current study is the possible spatial correlation of these sites with optimal routs calculated in QGIS for the two most likely alternatives of the usual route between different sites, one of this with E-W orientation, and the other one with an N-S orientation. This analysis has not been completed yet.

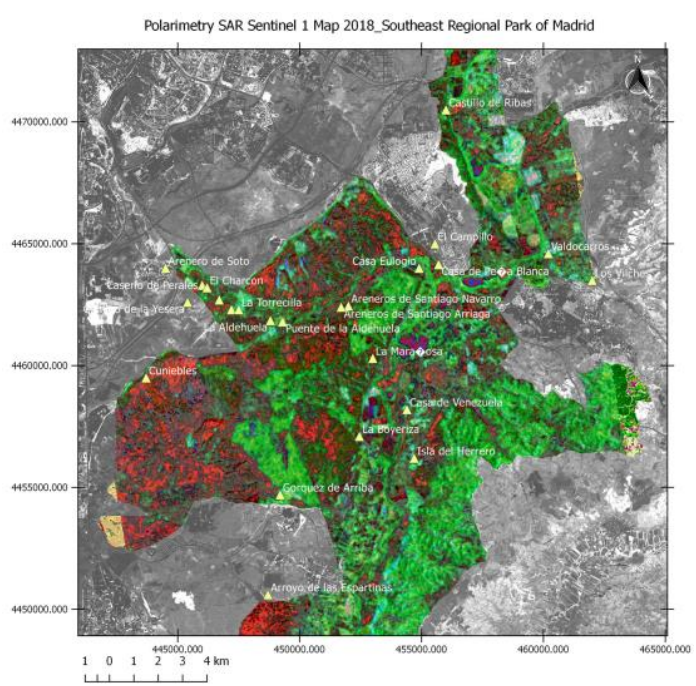

Figure 3. SAR polarimetry mapping from Sentinel 1 (NDVI background) and relation shift with cultural heritage in the Jarama Valley.

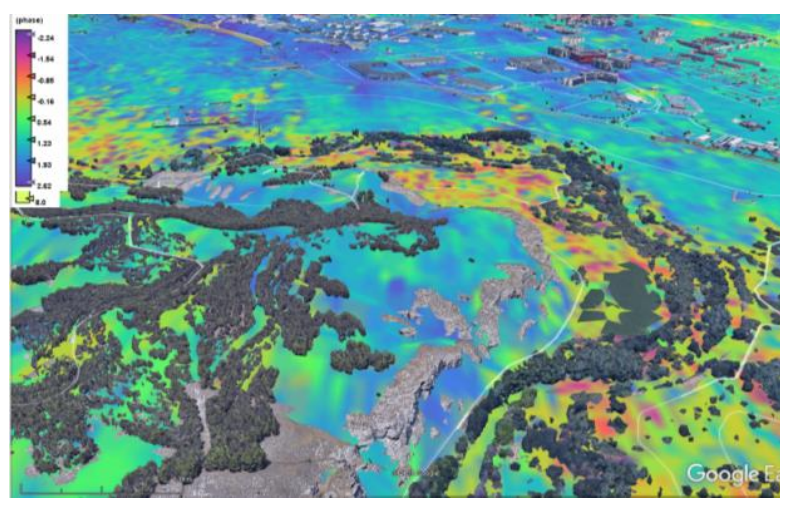

Figure 4. 3D SAR differential interferometry from Sentinel 1 confirming deformation cultural heritage in the Jarama Valley (source: Google Earth).
The results obtained from the multispectral analysis from Sentinel 2 and ATM have been integrated with the processed SAR data.

The responses from the surfaces with humidity concentration in Sentinel 1 images correspond to those detected in the ATM images and those that can be deduced from vegetation and soil indexes calculated for the Southeast Regional Park.

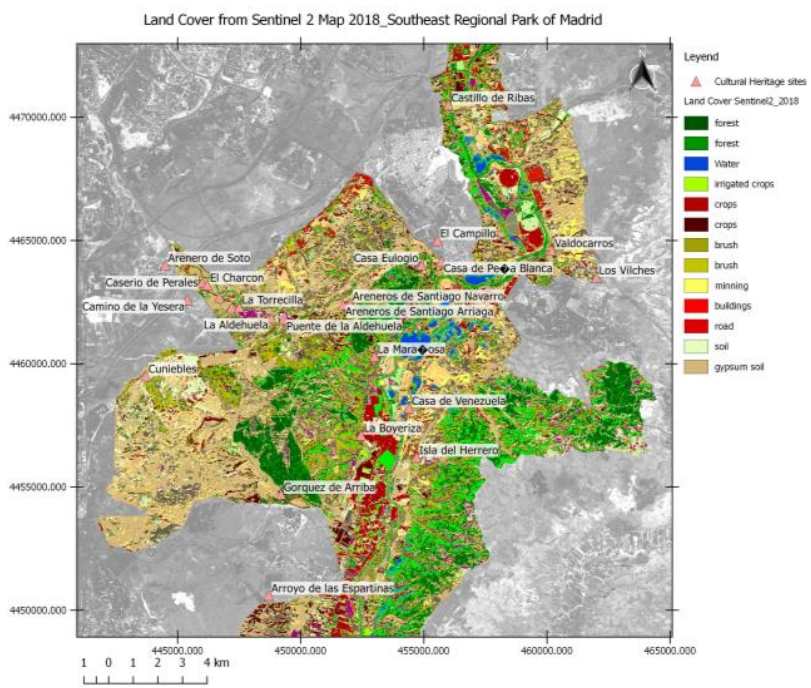

Figure 5. Land cover mapping from Sentinel 2 (NDVI background) and relation shift with cultural heritage in the Jarama Valley.

In this research, anomalies obtained for the standard method RX (Red and Xiaoli, 1990; Malpica et al. 2008) have been verified by those computed using methods based on subspaces, as the Subspace RX (SSRX) and the Orthogonal Subspace Projection (OSPRX) (Borghys et al., 2012; Nasrabadi, 2008).

The computation in all methods has been carried out separately for spectral ranges of reflective channels and emissive for ATM test data set.

The main challenge is how to characterize "interestingness" in a numerical fashion. In the case of this paper "interestingness" can be defined in terms of outliers. In this sense, we have been calculated a thermal index (Rejas et al., 2009), profiting from the separability between diagnostic bands in the emissive spectrum.

The relationship between the spectral anomalies and the diagnostic bands of the heritage materials has been studied.

It has been established a clear relationship between thermal anomalies and particular heritage remains and materials, as ancient buildings, walls, stones, as well as a clear relationship between VNIR anomalies and heritage materials with a great influence of the diagnostics bands in SWIR spectrum (for example tiles, stone walls, clay walls, trenches or buried remains).

The relationship between spectral patterns and anomalies has been recognized. Thermal anomalies in the Jarama Valley are corresponding to geometric patterns and linear 
(Rejas and Burillo, 2016), most of them with an extraction and a very quick interpretation.
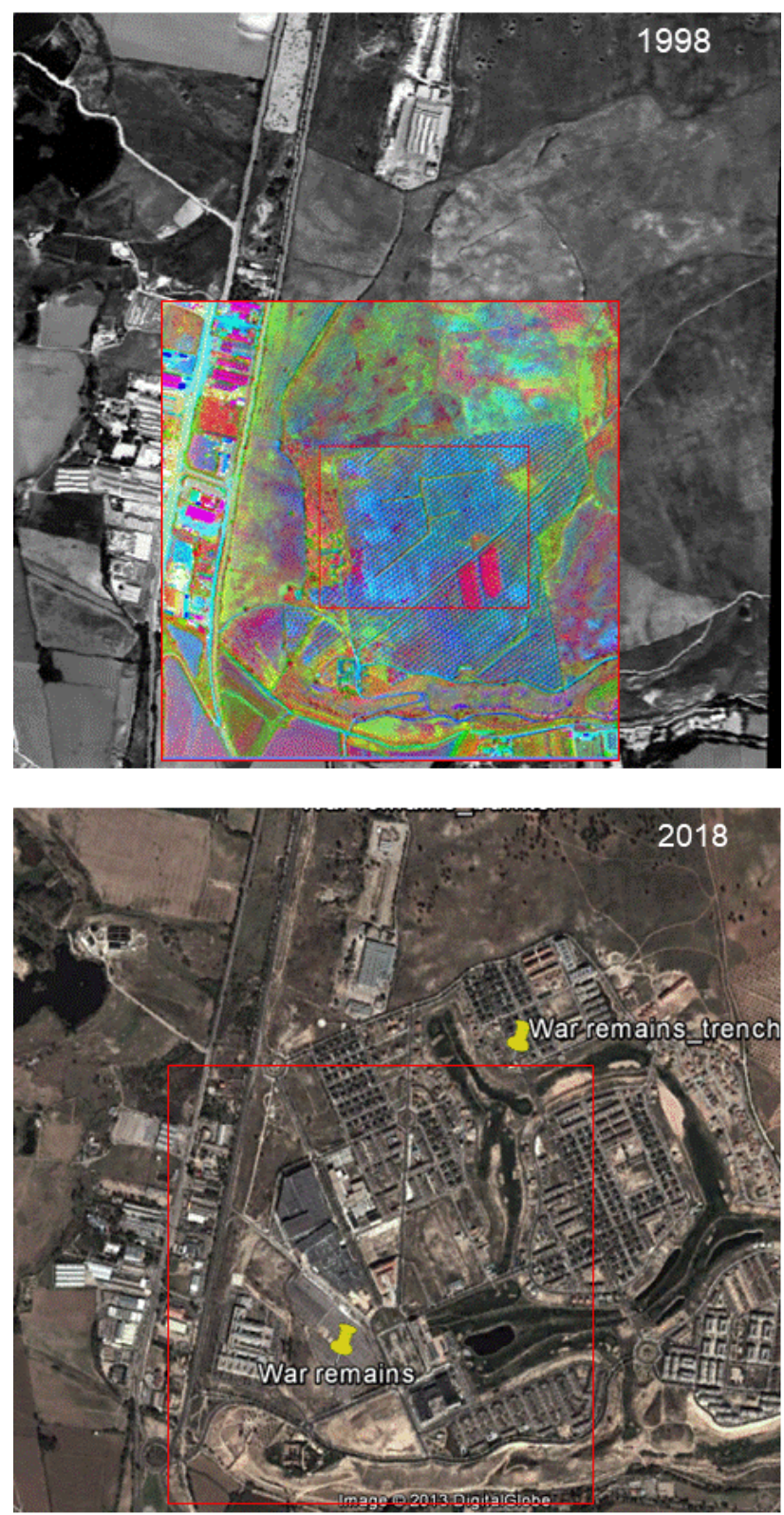

Figure 5. Spectral anomalies from ATM data validating war heritage (up) and change detection between 1998 and 2018 (down) in the Jarama Valley (source: Google Earth).

\section{Conclusions}

An approach of multi remote sensing methods has been applied to improve heritage mapping in the Jarama Valley. The characteristics of high-resolution data in the reflective, emissive and microwave range, for heritage sites in the Southeast Regional Park of Madrid has been studied using Sentinel 1 and Sentinel 2 from the European program Copernicus, and airborne ATM test data sets. This paper evaluates the performance of detection methods in scenes with different heritage backgrounds.

It has been observed that the thermal and SAR results, anomalies and interferogram, respectively, of particular surfaces can help to characterize the types of patterns associated with buried heritage structures. Higher concentrations of some heritage materials (manmade structures, clay walls, stonewalls, trenches, etc.), in scenarios where the sources of error are minimized, are correlated with the anomalies in the VNIR range.

This approach could improve the remote sensing cartography processes, searching areas and automatically detecting or conforming heritage materials of interest by virtue of their surface characteristics.

\section{Acknowledgements}

This work has been developed within the framework of the R\&D projects: I+D+i: HAR2015-68032-P titled La Serranía Celtibérica y Segeda, El Patrimonio Histórico como motor de Desarrolllo Rural, financed by MEC and FEDER funds.

\section{References}

Berni, J.A., Zarco-Tejada, P., Suárez, L and Fereres, E., (2010). Thermal and narrowband multispectral remote sensing for vegetation monitoring from an Unmanned Aerial Vehicle. IEEE Transactions On Geoscience And Remote Sensing, Vol. 47, No. 3.

Borghys, D., Achard, V., Kasen, I. and Perneel, C. (2012). Comparative evaluation of hyperspec-tral anomaly detection methods in scenes with diverse complexity. In Proc. OPTRO2012 Symposium on Optronics in Defence and Security, Paris, France.

Cocks, T., Jenssen, A., Stewart, I., Wilson and Shields, T. (1998). The HyMAP Airborne Hyperspectral Sensor: The System, Calibration and Performance. Proc. 1st EARSeL Workshop on Imaging Spectroscopy (M. Schaepman, D. Schläpfer, and K.I. Itten, Eds. Zurich, EARSeL, Paris, p. 37- 43.

Farjas, M., Rejas, J.G., Gómez, J.A., De Miguel, E. and Fernández, A. (2003). Airborne Multispectral Remote Sensing Application in Archaeological Areas. The Eway into the four Dimensions of Cultural Heritage, CAA Congress, Vienna City Hall.

Haboudane, D., Miller, J.R., Tremblay, N., Zarco-Tejada, P.J. and Dextraze, T., (2002). Integrated narrow-band vegetation indices for prediction of crop chlorophyll content for application to precision agriculture. Remote Sensing of Environment. vol. 81, no. 2/3, pp. 416-426.

Malpica, J.A., Rejas, J.G. and Alonso, M.C. (2008). A projection pursuit algorithm for anomaly detection in hyperspectral imagery. Pattern Recognition 41 (11) pp: 3313-3327, 2008. ISSN 0031-3203.

Nasrabadi, N.M. (2008). Multisensor Joint Fusion and Detection of Mines Using SAR and Hyperspectral. Proceedings of IEEE Sensors Conference.

Reed, I.S. (1990) and Xiaoli, Y. (1990). Adaptative multiple-band CFAR detection of an optical pattern with unknown spectral distribution. IEEE Transactions on Acoustics. Speech and Signal Processing, Vol. 38, No 10. 
Rejas, J.G. and Burillo, F., (2016). "Teledetección Aplicada a la Arqueología. Manual de Tecnologías de la Información Geográfica aplicadas a la Arqueología”. Ed. Comunidad de Madrid, Museo Arqueológico Regional: Ilustre Colegio Oficial de Doctores y Licenciados en Filosofía y Letras y en Ciencias de la Comunidad, pp 241-270, ISBN:978-84-9781-869-8, Madrid (España).

Rejas, J.G., Pineda, M.C., Véliz, S.V., Euraque, D., Martínez, E., Rodríguez, J.R. and Farjas, M. (2009). Archaeological remote sensing approach in Honduras. A project for cultural heritage and human habitats protection. 3th International Conference on Remote Sensing in Archaeology, Tiruchirapalli (India). BAR International Series 2118.

Rejas, J.G., Burillo, F., López, R., Cano, M.A., Sáiz, M.E. and Farjas, M. (2009). Integrating SAR data and hyperspectral analysis for the archaeological survey of the Segeda city, Spain. 3th International Conference on Remote Sensing in Archaeology, Tiruchirapalli (India). BAR International Series 2118.

Rondeaux, G., Steven, M. and Baret, F., (1996). Optimization of soil-adjusted vegetation indices. Remote Sening of Environment. vol. 55, no. 2, pp. 95107. 